\title{
DUALITY IN NONLINEAR FRACTIONAL PROGRAMMING PROBLEM USING FUZZY PROGRAMMING AND GENETIC ALGORITHM
}

\author{
Ananya Chakraborty \\ Assistant Professor, Department of Mathematics, Vemana Institute of Technology, \\ Kormangala $3^{\text {rd }}$ Block, Bangalore-34. Karnataka, India
}

\begin{abstract}
In this paper we have considered nonlinear fractional programming problem with multiple constraints. A pair of primal and dual for a special type of nonlinear fractional programming has been considered under fuzzy environment. Exponential membership function has been used to deal with the fuzziness. Duality results have been developed for the special type of nonlinear programming using exponential membership function. The method has been illustrated with numerical example. Genetic Algorithm as well as Fuzzy programming approach has been used to solve the problem.
\end{abstract}

\section{KEYWORDS}

Fuzzy Mathematical Programming, Nonlinear Fractional Programming, Exponential membership function, Decision Analysis, Genetic Algorithm.

\section{INTRODUCTION}

Several factors in the real world imply the increase in use of nonlinear programming models. There are several classes of problems where nonlinear programming had had a great impact for example oil and petrochemical industries, nonlinear network problems and economic planning models. The area where nonlinear programming can be used in these several classes of problems is given in detail in (Ladson et al., 1980). The concept of fuzzy programming in decision making problem was first proposed by (Bellmann and Zadeh, 1970). Many authors have applied fuzzy programming approach in different area of linear programming (Zimmermann, 1978, StancuMinasian et al., 1978, Chakraborty and Gupta, 2002). In (Jimenez, 2005), author has considered a non linear programming problem with fuzzy constraints and the solution has been obtained by muti objective evolutionary Algorithms. An interactive cutting plane algorithm for fuzzy multi objective nonlinear programming problems has been presented in (Kanaya, 2010). In (Jameel, 2012), author has obtained accurate results for solving non linear programming using fuzzy environment by using properties of fuzzy set and fuzzy number with linear membership function.

Fractional programs arise in management decision making as well as outside of it. They also occur sometimes indirectly in modeling where initially no ratio is involved. The efficiency of a system is sometimes characterized by a ratio of technical and/or economical terms. Maximizing system efficiency then leads to a fractional program. List of frequently occurring objectives are maximization of productivity, maximization of return on investment, maximization of return/risk, 
minimization of cost/time, maximization of output/input, Non-Economic Applications. There are a number of management science problems which indirectly give rise to a fractional program; see e.g. (Schaible, 1981, 1983, 1995). A new method has been given by (Borza et al. 2012) for solving the linear fractional programming problems with interval coefficients in the objective function.

An algorithm has been developed by (Saad et al., 2011) for multi objective integer nonlinear fractional programming problem under fuzziness. In order to defuzzify the problem, he has developed the concept of $\alpha$-level set of the fuzzy number is given and for obtaining an efficient solution to the problem (FMOINLFP), a linearization technique is presented to develop the solution algorithm. In the paper (Biswas and Bose, 2012) author presented a fuzzy programming procedure to solve nonlinear fractional programming problems in which the parameters involved in objective function are considered as fuzzy numbers.

The duality theory for nonlinear multiobjective optimization problems in the field of the optimization theory has intensively developed during the last decades. In (Rodder and Zimmermann, 1980), a generalization of maxmin and minmax problems in a fuzzy environment is presented and thereby a pair of fuzzy dual linear programming problems is constructed. An economic interpretation of this duality in terms of market and industry is also discussed in that paper. In (Bector and Chandra, 2002), a pair of linear programming primal-dual problem is introduced under fuzzy environment and appropriate results were proved to establish the duality relationship between them. In (Liu, 1995) a constructive approach has been proposed to duality for fuzzy multiple criteria and multiple constraint level linear programming problems. (Biswas and Bose, 2012) gives a parametric approach for the duality in fuzzy multi criteria and multi constraint level linear programming problem. In (Gupta and Mehlawat, 2009), a study of a pair of fuzzy primal-dual linear programming problems has been presented and calculated duality results using an aspiration level approach using exponential membership function, while a discussion of fuzzy primal dual linear programming problem with fuzzy coefficients has been presented in (Bector and Chandra, 2002; Liu, 1995). In (Bector and Chandra, 2002), a pair of linear programming primal-dual problem is introduced under fuzzy environment and appropriate results were proved to establish the duality relationship between them. Also in (Chakraborty et al. 2014), author has presented a pair of linear primal - dual programming using linear and exponential membership function using fuzzy programming approach and genetic algorithm approach.

In this paper we have extended the work done by (Gupta and Mehlawat , 2009) in which he has solved duality in convex fractional programming using linear membership function. We have taken the same kind of problem and proved the duality theorems considering exponential membership function for fuzzified objective function and constraints and then solved using LINGO and Genetic Algorithm also and the results have been analysed.

The paper has been organized as follows: In section 2, the nonlinear fractional programming problem and its dual has been defined. The fuzzified and the crisp formulation have been defined for the primal and dual. In section 3, the necessary duality results have been developed. The numerical example defined in (Gupta and Mehlawat, 2009) has been illustrated in section 4. Finally, analysis of the solution and concluding remarks has been presented in section 5 .

\section{NON LINEAR FRACTIONAL PROGRAMMING PROBLEM}

The nonlinear fractional programming problem (Gupta and Mehlawat, 2009) can be defined as: 
$\operatorname{Max} f(x)=\frac{\left(c^{t} x\right)^{2}}{d^{t} x}$

Subject to $A x \leq b$

$$
x \geq 0
$$

The dual form of the above problem can be written as

Min $g(u, v)=b^{t} u$

Subject to $A^{t} u+d v^{2} \geq 2 c v$

$$
u, v \geq 0
$$

Where $A$ is $m \times n$ matrix, $x, c, b$ are column vectors with $n$ components and $b$ is a column vector of $m$ components.

\subsection{Fuzzified Non Linear Fractional Programming Problem}

Fuzzified form of nonlinear fractional programming problem can be defined as

Find $x \in R^{n}$

$$
\begin{gathered}
\text { Subject to } f(x)=\frac{\left(c^{t} x\right)^{2}}{d^{t} x} \tilde{\geq} Z_{0} \\
A x \leq b \\
x \geq 0
\end{gathered}
$$

Where $Z_{0}$ is the aspiration level of the objective function of the primal problem. " $\tilde{\leq}$ " and " $\tilde{\geq}$ " denotes the flexibility of the objective function and the multi constraints.

Fuzzified form of the dual of the problem can be defined as

Find $u \in R^{m}$

$$
\begin{array}{cl}
\text { Subject to } & \mathrm{g}(\mathrm{u}, \mathrm{v})=u^{t} b \tilde{\leq} w_{0} \\
& A^{t} u+d v^{2} \tilde{\geq} 2 c v \\
u, v \geq 0
\end{array}
$$

Where $w_{0}$ is the aspiration level of the objective function of the dual problem.

\subsection{Crisp Formulations using Exponential Membership Function}

The advantage of nonlinear membership function over linear membership function is already described in (Gupta and Mehlawat, 2009; Chakraborty et al. 2014). Linear membership function is most commonly used in fuzzy linear programming problem because it is simple and it is defined by fixing two points. Also, linear membership function has been used in a vast range of nonlinear problems (Bector and Chandra, 2002; Gupta and Mehlawat, 2009; Chakraborty et al. 2014). But in many practical situations linear membership function is not a suitable 
International Journal of Soft Computing, Mathematics and Control (IJSCMC), Vol.4, No. 1, February 2015

representation. Furthermore, if the membership function is interpreted as the fuzzy utility of the decision maker, used for describing levels of indifference, preference towards uncertainty, then a nonlinear membership function provides a better representation than a linear membership function. Moreover, unlike linear membership function, for nonlinear membership functions the marginal rate of increase (or decrease) of membership values as a function of model parameters is not constant- a technique that reflects reality better than the linear case (Gupta and Mehlawat, 2009).

Let us consider the following form of exponential membership function (Gupta and Mehlawat , 2009; Chakraborty et al. 2014) for the fuzzy nonlinear fractional programming problem respectively.

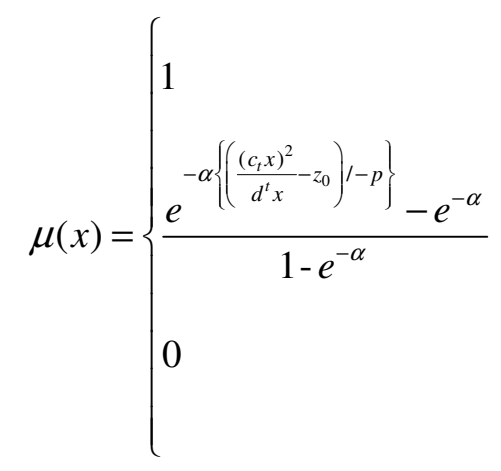

$$
\left.\begin{array}{c}
\text { if } \quad \frac{\left(\mathrm{c}_{t} x\right)^{2}}{d^{t} x} \geq z_{0} \\
\text { if } \mathrm{z}_{0}-p<\frac{\left(\mathrm{c}_{t} x\right)^{2}}{d^{t} x}<z_{0} \\
\text { if } \quad \frac{\left(\mathrm{c}_{t} x\right)^{2}}{d^{t} x} \leq z_{0}-p
\end{array}\right\}
$$

$\mu_{j}(x)=\left\{\begin{array}{l}1 \\ \frac{e^{-\alpha_{j}\left\{\left(b_{j}-A_{j} x\right) /-q_{j}\right\}}-e^{-\alpha_{j}}}{1-e^{-\alpha_{j}}} \\ 0\end{array}\right.$

$$
\left.\begin{array}{l}
\text { if } \quad A_{j} x \leq b_{j} \\
\text { if } \quad b_{j}<A_{j} x<b_{j}+q_{j} \\
\text { if } \quad A_{j} x \geq b_{j}+q_{j}
\end{array}\right\} \quad j=1,2, \ldots \ldots . m
$$

Where $\alpha$ and $\alpha_{j}$ are user defined parameters which determine the shape of the membership function. $p$ and $q_{j}(j=1,2, \ldots . . m)$ are subjectively chosen constant of admissible violations such that $p$ is associated with nonlinear fractional objective function and $q_{j}{ }^{\prime} s$ are associated with $\mathrm{m}$ linear constraints of the problem.

Therefore using exponential membership function the crisp formulation of the problem can be given by

$\operatorname{Max} \lambda$

$$
\begin{aligned}
\text { Subject to } & \lambda \leq \frac{e^{-\alpha\left\{\left(\frac{\left(c_{t} x\right)^{2}}{d^{t} x}-z_{0}\right) /-p\right\}}-e^{-\alpha}}{1-e^{-\alpha}} \\
& \lambda \leq \frac{e^{-\alpha_{j}\left\{\left(b_{j}-A_{j} x\right) /-q_{j}\right\}}-e^{-\alpha_{j}}}{1-e^{-\alpha_{j}}} \quad j=1,2, \ldots m \\
& \lambda \leq 1, \lambda \geq 0, x \geq 0 .
\end{aligned}
$$


International Journal of Soft Computing, Mathematics and Control (IJSCMC), Vol.4, No. 1, February 2015

or $\operatorname{Max} \lambda$

Subject to $p \log \left\{\lambda\left(1-e^{-\alpha}\right)+e^{-\alpha}\right\} \leq \alpha\left(\frac{\left(c_{t} x\right)^{2}}{d^{t} x}-z_{0}\right)$

$$
\begin{gathered}
q_{j} \log \left\{\lambda\left(1-e^{-\alpha_{j}}\right)+e^{-\alpha_{j}}\right\} \leq \alpha_{j}\left(b_{j}-A_{j} x\right) \quad, j=1,2, \ldots m \\
\lambda \leq 1, \lambda \geq 0, x \geq 0 .
\end{gathered}
$$

The exponential membership function for the dual problem objective function and nonlinear constraints are given by

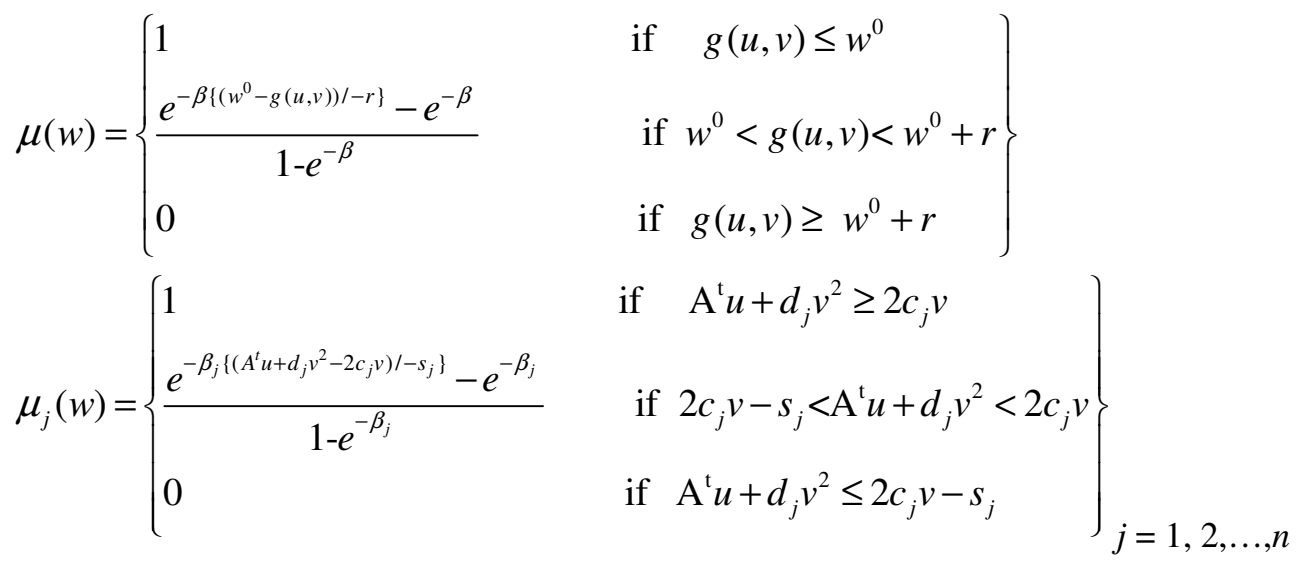

Where $\beta$ and $\beta_{j}$ 's are user defined parameters which determine the shape of the membership function. $r$ and $s_{j}(j=1,2, \ldots \ldots, n)$ are subjectively chosen constant of admissible violations such that $r$ is associated with objective function and $s_{j}{ }^{\prime} s$ are associated with $n$ linear constraints of the dual problem.

$\operatorname{Min}(-\eta)$

Subject to $\eta \leq \frac{e^{-\beta\left\{\left(w^{0}-g(u, v)\right) /-r\right\}}-e^{-\beta}}{1-e^{-\beta}}$

$$
\begin{aligned}
& \eta \leq \frac{e^{-\beta_{j}\left\{\left(A^{t} u+d_{j} \nu^{2}-2 c_{j} v\right) /-s_{j}\right\}}-e^{-\beta_{j}}}{1-e^{-\beta_{j}}} \quad j=1,2, \ldots n \\
& \eta \leq 1, \eta \geq 0, u, v \geq 0 .
\end{aligned}
$$

or $\operatorname{Min}(-\eta)$

Subject to $\quad r \log \left\{\eta\left(1-e^{-\beta}\right)+e^{-\beta}\right\} \leq \beta\left(w^{0}-g(u, v)\right)$ 
International Journal of Soft Computing, Mathematics and Control (IJSCMC), Vol.4, No. 1, February 2015

$$
\begin{aligned}
& s_{j} \log \left\{\eta\left(1-e^{-\beta_{j}}\right)+e^{-\beta_{j}}\right\} \leq \beta_{j}\left(A_{j}^{t} u+d_{j} v^{2}-2 c_{j} v\right) \quad, j=1,2, \ldots n \\
& \eta \leq 1, \eta \geq 0, u, v \geq 0 .
\end{aligned}
$$

\section{THEOREMS ON DUALITY}

We shall prove some duality theorems. In the theorems, $Q=\left(q_{1}, q_{2}, \ldots \ldots, q_{m}\right), S=\left(s_{1}, s_{2}, \ldots \ldots, s_{n}\right)$ are column vectors

Theorem 3.1 (Modified fuzzy weak duality theorem): Let $(x, \lambda)$ be the feasible solution of crisp primal problem (7) and $(u, v, \eta)$ be feasible solution of crisp dual problem (10). Then

$$
\sum_{i=1}^{m} \frac{\log \left\{\lambda\left(1-e^{-\alpha_{i}}\right)+e^{-\alpha_{i}}\right\}}{\alpha_{i}} u^{t} Q+\sum_{j=1}^{n} \frac{\log \left\{\eta\left(1-e^{-\beta_{j}}\right)+e^{-\beta_{j}}\right\}}{\beta_{j}} S^{t} x \tilde{\leq} u^{t} b-\frac{\left(c^{t} x\right)^{2}}{d^{t} x}
$$

Proof: Since $(x, \lambda)$ be the feasible solution of crisp primal problem $(7)$ and $(w, \eta)$ be feasible solution of crisp dual problem (10), then

$$
\begin{aligned}
& q_{i} \log \left\{\lambda\left(1-e^{-\alpha_{i}}\right)+e^{-\alpha_{i}}\right\} \leq \alpha_{i}\left(b_{i}-A_{i} x\right), i=1,2, \ldots m \\
& s_{j} \log \left\{\eta\left(1-e^{-\beta_{j}}\right)+e^{-\beta_{j}}\right\} \leq \beta_{j}\left(A_{j}^{t} u+d_{j} v^{2}-2 c_{j} v\right), j=1,2, \ldots n \\
& \text { Or } \sum_{i=1}^{m} \frac{\log \left\{\lambda\left(1-e^{-\alpha_{i}}\right)+e^{-\alpha_{i}}\right\}}{\alpha_{i}} Q \leq b-A x \\
& \quad \sum_{j=1}^{n} \frac{\log \left\{\eta\left(1-e^{-\beta_{j}}\right)+e^{-\beta_{j}}\right\}}{\beta_{j}} S \leq A^{t} u+d v^{2}-2 c v
\end{aligned}
$$

Multiplying the equation (11) by transpose of $u$ and taking transpose of equation (12) and multiplying equation by $x$, we have

$$
\begin{aligned}
& \sum_{i=1}^{m} \frac{\log \left\{\lambda\left(1-e^{-\alpha_{i}}\right)+e^{-\alpha_{i}}\right\}}{\alpha_{i}} u^{t} Q \leq u^{t} b-u^{t} A x \\
& \sum_{j=1}^{n} \frac{\log \left\{\eta\left(1-e^{-\beta_{j}}\right)+e^{-\beta_{j}}\right\}}{\beta_{j}} S^{t} x \leq u^{t} A x+v^{t^{2}} d^{t} x-2 v^{t} c^{t} x
\end{aligned}
$$

Adding the above two inequalities, we have

$$
\begin{aligned}
& \sum_{i=1}^{m} \frac{\log \left\{\lambda\left(1-e^{-\alpha_{i}}\right)+e^{-\alpha_{i}}\right\}}{\alpha_{i}} u^{t} Q+\sum_{j=1}^{n} \frac{\log \left\{\eta\left(1-e^{-\beta_{j}}\right)+e^{-\beta_{j}}\right\}}{\beta_{j}} S^{t} x \leq u^{t} b+v^{t^{2}} d^{t} x-2 v^{t} c^{t} x \\
& \operatorname{Or} \sum_{i=1}^{m} \frac{\log \left\{\lambda\left(1-e^{-\alpha_{i}}\right)+e^{-\alpha_{i}}\right\}}{\alpha_{i}} u^{t} Q+\sum_{j=1}^{n} \frac{\log \left\{\eta\left(1-e^{-\beta_{j}}\right)+e^{-\beta_{j}}\right\}}{\beta_{j}} S^{t} x \leq u^{t} b+\left\{\left(d^{t} x\right)^{1 / 2} v^{t}-\frac{\left(c^{t} x\right)}{\left(d^{t} x\right)^{1 / 2}}\right\}^{2}-\frac{\left(c^{t} x\right)^{2}}{d^{t} x}
\end{aligned}
$$


International Journal of Soft Computing, Mathematics and Control (IJSCMC), Vol.4, No. 1, February 2015

$$
\begin{aligned}
& \sum_{i=1}^{m} \frac{\log \left\{\lambda\left(1-e^{-\alpha_{i}}\right)+e^{-\alpha_{i}}\right\}}{\alpha_{i}} u^{t} Q+\sum_{j=1}^{n} \frac{\log \left\{\eta\left(1-e^{-\beta_{j}}\right)+e^{-\beta_{j}}\right\}}{\beta_{j}} S^{t} x \leq\left\{u^{t} b-\frac{\left(c^{t} x\right)^{2}}{d^{t} x}\right\}+\left\{\left(d^{t} x\right)^{1 / 2} v-\frac{\left(c^{t} x\right)}{\left(d^{t} x\right)^{1 / 2}}\right\}^{2} \\
& \sum_{i=1}^{m} \frac{\log \left\{\lambda\left(1-e^{-\alpha_{i}}\right)+e^{-\alpha_{i}}\right\}}{\alpha_{i}} u^{t} Q+\sum_{j=1}^{n} \frac{\log \left\{\eta\left(1-e^{-\beta_{j}}\right)+e^{-\beta_{j}}\right\}}{\beta_{j}} S^{t} x \leq\left\{u^{t} b-\frac{\left(c^{t} x\right)^{2}}{d^{t} x}\right\}
\end{aligned}
$$

Remark: When $\lambda=1, \eta=1$ in the above inequality we get the objective function of primal problem is imprecisely less than the objective function of the dual $\left\{u^{t} b \tilde{\leq} \frac{\left(c^{t} x\right)^{2}}{d^{t} x}\right\}$ which can be defined as the standard fuzzified weak duality theorem in a multi objective linear programming problem.

Theorem 3.2: Let $(\hat{x}, \hat{\lambda})$ be feasible solution of crisp primal problem $(7)$ and $(\hat{w}, \hat{\eta})$ be feasible solution of crisp dual problem (10) such that

$$
\begin{aligned}
& \sum_{i=1}^{m} \frac{\log \left\{\hat{\lambda}\left(1-e^{-\alpha_{i}}\right)+e^{-\alpha_{i}}\right\}}{\alpha_{i}} \hat{w}^{T} Q+\sum_{j=1}^{n} \frac{\log \left\{\hat{\eta}\left(1-e^{-\beta_{j}}\right)+e^{-\beta_{j}}\right\}}{\beta_{j}} S^{T} \hat{x}=\hat{u^{t}} b-\frac{\left(c^{t} \hat{x}\right)^{2}}{d^{t} \hat{x}} \\
& \frac{\log \left\{\hat{\lambda}\left(1-e^{-\alpha}\right)+e^{-\alpha}\right\}}{\alpha} p+\frac{\log \left\{\hat{\eta}\left(1-e^{-\beta}\right)+e^{-\beta}\right\}}{\beta} r=\left(\frac{\left(c^{t} \hat{x}\right)^{2}}{d^{t} \hat{x}}-\hat{u^{t} b}\right)+\left(w_{0}-z_{0}\right)
\end{aligned}
$$

(iii) The aspiration levels $z_{0}$ and $w_{0}$ satisfy $\left(z_{0}-w_{0}\right) \leq 0$

Then $(\hat{x}, \hat{\lambda})$ is optimal solution of primal problem and $(\hat{w}, \hat{\eta})$ be optimal solution of dual problem.

Proof: Let $(x, \lambda)$ be feasible solution of crisp primal problem $(7)$ and $(w, \eta)$ be feasible solution of crisp dual problem (10). Then from Theorem 3 we have,

$$
\sum_{i=1}^{m} \frac{\log \left\{\lambda\left(1-e^{-\alpha_{i}}\right)+e^{-\alpha_{i}}\right\}}{\alpha_{i}} u^{t} Q+\sum_{j=1}^{n} \frac{\log \left\{\eta\left(1-e^{-\beta_{j}}\right)+e^{-\beta_{j}}\right\}}{\beta_{j}} S^{t} x-\left(u^{t} b-\frac{\left(c^{t} x\right)^{2}}{d^{t} x}\right) \tilde{\leq} 0
$$

(13)

From condition (i) and Equation (13) we have

$$
\begin{aligned}
& \sum_{i=1}^{m} \frac{\log \left\{\lambda\left(1-e^{-\alpha_{i}}\right)+e^{-\alpha_{i}}\right\}}{\alpha_{i}} u^{t} Q+\sum_{j=1}^{n} \frac{\log \left\{\eta\left(1-e^{-\beta} j\right)+e^{-\beta} j\right\}}{\beta_{j}} S^{t} x-\left(u^{t} b-\frac{\left(c^{t} x\right)^{2}}{d^{t} x}\right) \\
& \tilde{\leq} \sum_{i=1}^{m} \frac{\log \left\{\hat{\lambda}\left(1-e^{-\alpha_{i}}\right)+e^{-\alpha_{i}}\right\}}{\alpha_{i}} \hat{u}^{t} Q+\sum_{j=1}^{n} \frac{\log \left\{\hat{\eta}\left(1-e^{-\beta_{j}}\right)+e^{-\beta_{j}}\right\}}{\beta_{j}} S^{t} \hat{x}-\left(\hat{u^{t}} b-\frac{\left(c^{t} \hat{x}\right)^{2}}{d^{t} \hat{x}}\right)
\end{aligned}
$$


International Journal of Soft Computing, Mathematics and Control (IJSCMC), Vol.4, No. 1, February 2015

Thus, $(\hat{x}, \hat{\lambda}, \hat{w}, \hat{\eta})$ is optimal to the problem whose maximum objective value is 0 .

$\operatorname{Max} \sum_{i=1}^{m} \frac{\log \left\{\lambda\left(1-e^{-\alpha_{i}}\right)+e^{-\alpha_{i}}\right\}}{\alpha_{i}} u^{t} Q+\sum_{j=1}^{n} \frac{\log \left\{\eta\left(1-e^{-\beta} j\right)+e^{-\beta} j\right\}}{\beta_{j}} S^{t} x-\left(u^{t} b-\frac{\left(c^{t} x\right)^{2}}{d^{t} x}\right)$

Subject to

$$
\begin{aligned}
& p \log \left\{\lambda\left(1-e^{-\alpha}\right)+e^{-\alpha}\right\} \leq \alpha\left(\frac{\left(c^{t} x\right)^{2}}{d^{t} x}-Z_{0}\right) \\
& q_{i} \log \left\{\lambda\left(1-e^{-\alpha_{i}}\right)+e^{-\alpha_{i}}\right\} \leq \alpha_{i}\left(b_{i}-A_{i} x\right) \\
& r \log \left\{\eta\left(1-e^{-\beta}\right)+e^{-\beta}\right\} \leq \beta\left(w_{0}-u^{t} b\right) \\
& s_{j} \log \left\{\eta\left(1-e^{-\beta_{j}}\right)+e^{-\beta_{j}}\right\} \leq \beta_{j}\left(A_{j}^{t} u+d_{j} v^{2}-2 c_{j} v\right) \\
& \lambda \leq 1, \lambda \geq 0, x \geq 0, \eta \leq 1, u, v \geq 0, \eta \geq 0 .
\end{aligned} \quad(i=1,2, \ldots ., m)
$$

Adding condition (i) and (ii), we have

$$
\begin{aligned}
& \sum_{i=1}^{m} \frac{\log \left\{\lambda\left(1-e^{-\alpha_{i}}\right)+e^{-\alpha_{i}}\right\}}{\alpha_{i}} u^{t} Q+\sum_{j=1}^{n} \frac{\log \left\{\eta\left(1-e^{-\beta} j\right)+e^{-\beta} j\right\}}{\beta_{j}} S^{t} x-\left(u^{t} b-\frac{\left(c^{t} x\right)^{2}}{d^{t} x}\right)+ \\
& \frac{\log \left\{\hat{\lambda}\left(1-e^{-\alpha}\right)+e^{-\alpha}\right\}}{\alpha} p+\frac{\log \left\{\hat{\eta}\left(1-e^{-\beta}\right)+e^{-\beta}\right\}}{\beta} r-\left(\frac{\left(c^{t} \hat{x}\right)^{2}}{d^{t} \hat{x}}-u^{t} b\right)-\left(w_{0}-z_{0}\right)=0
\end{aligned}
$$

Or

$$
\begin{aligned}
& \sum_{i=1}^{m} \frac{\log \left\{\lambda\left(1-e^{-\alpha_{i}}\right)+e^{-\alpha_{i}}\right\}}{\alpha_{i}} u^{t} Q+\sum_{j=1}^{n} \frac{\log \left\{\eta\left(1-e^{-\beta} j\right)+e^{-\beta} j\right\}}{\beta j} S^{t} x+ \\
& \frac{\log \left\{\hat{\lambda}\left(1-e^{-\alpha}\right)+e^{-\alpha}\right\}}{\alpha} p+\frac{\log \left\{\hat{\eta}\left(1-e^{-\beta}\right)+e^{-\beta}\right\}}{\beta} r+\left(z_{0}-w_{0}\right)=0
\end{aligned}
$$

Each term in the above sum is non positive as $\hat{\lambda}, \hat{\eta} \leq 1$.

Therefore, $\sum_{i=1}^{m} \frac{\log \left\{\hat{\lambda}\left(1-e^{-\alpha_{i}}\right)+e^{-\alpha_{i}}\right\}}{\alpha_{i}} \hat{u}^{t} Q=0$

$$
\begin{aligned}
& \sum_{j=1}^{n \times t} \frac{\log \left\{\hat{\eta}\left(1-e^{-\beta_{j}}\right)+e^{-\beta_{j}}\right\}}{\beta_{j}} S^{t} \hat{x}=0 \\
& \frac{\log \left\{\hat{\lambda}\left(1-e^{-\alpha}\right)+e^{-\alpha}\right\}}{\alpha} p=0
\end{aligned}
$$


International Journal of Soft Computing, Mathematics and Control (IJSCMC), Vol.4, No. 1, February 2015

$$
\begin{aligned}
& \frac{\log \left\{\hat{\eta}\left(1-e^{-\beta}\right)+e^{-\beta}\right\}}{\beta} r=0 \\
& z_{0}-w_{0}=0
\end{aligned}
$$

Since $\frac{\log \left\{\lambda\left(1-e^{-\alpha}\right)+e^{-\alpha}\right\}}{\alpha} p \leq 0$ and $\frac{\log \left\{\eta\left(1-e^{-\beta}\right)+e^{-\beta}\right\}}{\beta} r \leq 0$, because $\hat{\lambda}, \hat{\eta} \leq 1$, we get

$\frac{\log \left\{\lambda\left(1-e^{-\alpha}\right)+e^{-\alpha}\right\}}{\alpha} p \leq \frac{\log \left\{\hat{\lambda}\left(1-e^{-\alpha}\right)+e^{-\alpha}\right\}}{\alpha} p$

$\frac{\log \left\{\eta\left(1-e^{-\beta}\right)+e^{-\beta}\right\}}{\beta} r \leq \frac{\log \left\{\hat{\eta}\left(1-e^{-\beta}\right)+e^{-\beta}\right\}}{\beta} r$

or $\lambda \leq \hat{\lambda}$ and $\eta \leq \hat{\eta}$

Thus, $(\hat{x}, \hat{\lambda})$ is optimal solution of (7) and $(\hat{w}, \hat{\eta})$ be optimal solution of (10).

\section{NUMERICAL EXAMPLE}

Let us consider the same numerical example which is defined in [20]. The primal dual nonlinear fractional programming problem can be defined as

$\operatorname{Min} f(x)=\frac{\left(2 x_{1}+x_{2}\right)^{2}}{x_{1}+2 x_{2}}$

Subject to $2 x_{1}+x_{2} \geq 6$

$$
\begin{aligned}
& x_{1}+3 x_{2} \geq 8 \\
& x_{1}, x_{2} \geq 0
\end{aligned}
$$

$\operatorname{Max} g(u, v)=6 u_{1}+8 u_{2}$

Subject to $2 u_{1}+u_{2}+v^{2}-4 v \leq 0$

$$
\begin{aligned}
& u_{1}+3 u_{2}+2 v^{2}-2 v \leq 0 \\
& u_{1}, u_{2}, v \geq 0
\end{aligned}
$$

The fuzzified nonlinear fractional programming problem can be written as:

Find $x \in R^{n}$

Min

Subject to $f(x)=\frac{\left(2 x_{1}+x_{2}\right)^{2}}{x_{1}+2 x_{2}} \stackrel{\sim}{\leq} 1$

$$
2 x_{1}+x_{2} \tilde{\geq} 6
$$


International Journal of Soft Computing, Mathematics and Control (IJSCMC), Vol.4, No. 1, February 2015

$$
\begin{aligned}
& x_{1}+3 x_{2} \geq 8 \\
& x_{1}, x_{2} \geq 0
\end{aligned}
$$

$\operatorname{Max} g(u, v)=6 u_{1}+8 u_{2} \tilde{\geq} 1$

Subject to $2 u_{1}+u_{2}+v^{2}-4 v \leq 0$

$$
\begin{aligned}
& u_{1}+3 u_{2}+2 v^{2}-2 v \tilde{\leq} 0 \\
& u_{1}, u_{2}, v \geq 0
\end{aligned}
$$

Let us take $p=2, q_{1}=1, q_{2}=1, \alpha=2, \alpha_{1}=2, \alpha_{2}=1$ for primal problem. Using exponential membership function, the crisp model of (14) can be written as:

$\operatorname{Min}-\lambda$

Subject to

$$
\begin{aligned}
& 4 x_{1}^{2}+x_{2}^{2}+4 x_{1} x_{2} \geq\left(x_{1}+2 x_{2}\right) \log (0.865 \lambda+0.1353) \\
& 4 x_{1}+2 x_{2}-12 \geq \log (0.865 \lambda+0.1353) \\
& x_{1}+3 x_{2}-8 \geq \log (0.865 \lambda+0.1353) \\
& \lambda \leq 1, \lambda \geq 0 \\
& x_{1}, x_{2} \geq 0
\end{aligned}
$$

Let us take $r=1, s_{1}=1, s_{2}=1, \beta=2, \beta_{1}=2, \beta_{2}=1$ for dual problem (15). Using exponential membership function, the crisp model can be written as:

Max $\eta$

Subject to

$$
\begin{aligned}
& \log (0.865 \eta+0.1353) \leq 12 u_{1}+16 u_{2}-2 \\
& \log (0.865 \eta+0.1353) \leq-4 u_{1}-2 u_{2}-2 v^{2}+8 v \\
& \log (0.865 \eta+0.1353) \leq-u_{1}-3 u_{2}-2 v^{2}+2 v \\
& 0 \leq \eta \leq 1, u_{1}, u_{2}, v \geq 0
\end{aligned}
$$

\section{SOLUTION AND ANALYSIS}

Using LINGO software, the solution of the primal problem (16) is given by

Local optimal solution found.

Objective value:

Infeasibilities:

$-1.000000$

Extended solver steps:

0.000000

Total solver iterations:

Model Class:

Total variables:

5
25
NLP
3
3
0

$\begin{array}{ll}\text { Nonlinear variables: } & 3 \\ \text { Integer variables: } & 0\end{array}$ 
International Journal of Soft Computing, Mathematics and Control (IJSCMC), Vol.4, No. 1, February 2015

Total constraints:

Nonlinear constraints: 3

Total nonzeros:

Nonlinear nonzeros:

11

Variable Value

$\begin{array}{cl}\text { LAMBDA } & 1.000000 \\ \mathrm{X} 1 & 1.234568 \\ \mathrm{X} 2 & 0.1000000 \mathrm{E}+08\end{array}$

Minimum value of objective function is -1 .

The solution of the dual problem (17) is given by

Local optimal solution found.

Objective value:

Infeasibilities:

Extended solver steps:

Total solver iterations:

Model Class:

Total variables:

Nonlinear variables:

Integer variables:

Total constraints:

Nonlinear constraints:

Total nonzeros:

Nonlinear nonzeros:

Variable Value

ETA $\quad 1.000000$

U1 0.1037300

U2 $\quad 0.4665636 \mathrm{E}-01$

$\mathrm{V} \quad 0.1359680$
1.000000

0.000000

5

37

NLP

4

2

0

5

3

13

5

Maximum value of the objective function is 1 .

Genetic Algorithm gives global optimal solution. The NSGA used here is a real parameter GA that works directly with the parameter values. NSGA (Nondominated Sorting Genetic Algorithm) uses nitching, as well as nondominated sorting of the solutions in every generation to ensure that the "good solutions get preference in selection for procreation". The non-dominated sorting GA uses a ranking selection method to emphasize good solutions and then a nitche building procedure to maintain a stable sub population of good solutions. Since multi objective GAs can find multiple pareto optimal solutions in one single run, the proposed technique is capable of finding multiple solutions to the problems.

The parameters used to solve the problem (16) in Genetic Algorithm are as follows:

Number of objective functions : 1 
International Journal of Soft Computing, Mathematics and Control (IJSCMC), Vol.4, No. 1, February 2015

Objective function \# 1 : Minimize

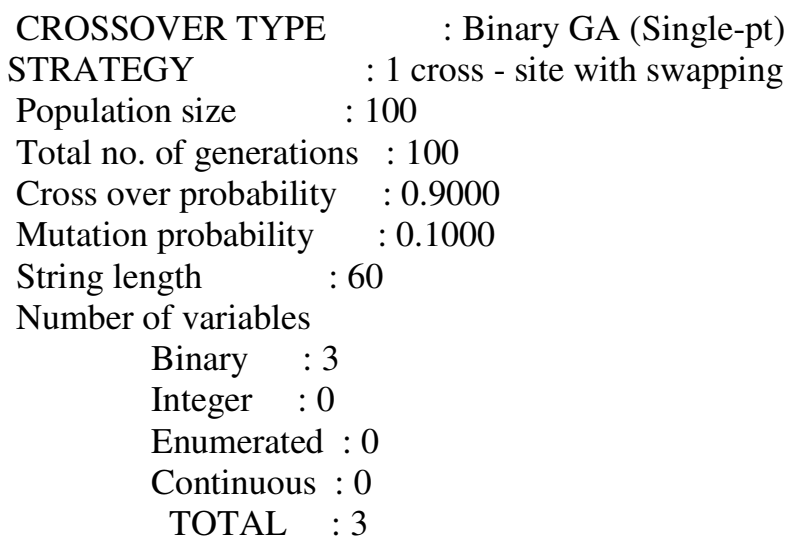

Epsilon for closeness : 0

Sigma-share value $: 0.2320$

Sharing Strategy : sharing on Parameter Space

Lower and Upper bounds :

$0.0000<=\lambda<=1.0000$

$0.0000<=x_{1}<=2.0000$

$0.0000<=x_{2}<=2.0000$

Table 1 gives a set of solution of problem (16).

Table 1: Primal Solution

\begin{tabular}{ccc}
\hline $\boldsymbol{\lambda}$ & $\boldsymbol{x}_{\boldsymbol{1}}$ & $\boldsymbol{x}_{\boldsymbol{2}}$ \\
\hline $\mathbf{0 . 9 4 2}$ & 1.952 & 1.946 \\
$\mathbf{0 . 9 9 2}$ & 1.673 & 1.976 \\
$\mathbf{0 . 9 6 2}$ & 1.794 & 1.974 \\
$\mathbf{0 . 9 4 2}$ & 1.884 & 1.916 \\
$\mathbf{0 . 9 7 7}$ & 1.514 & 1.922 \\
\hline
\end{tabular}

The parameters used to solve the problem (17) in Genetic Algorithm are as follows:

Number of objective functions : 1

Objective function \# 1 : Maximize

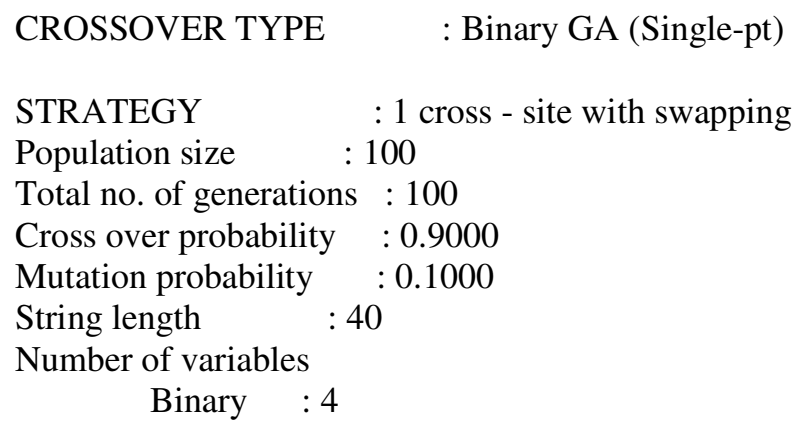


International Journal of Soft Computing, Mathematics and Control (IJSCMC), Vol.4, No. 1, February 2015

Integer : 0
Enumerated $: 0$
Continuous $: 0$
TOTAL $: 4$

Epsilon for closeness : 0

Sigma-share value $: 0.2810$

Sharing Strategy : sharing on Parameter Space

Lower and Upper bounds :

$0.0000<=\mathrm{u}_{1}<=2.0000$

$0.0000<=\mathrm{u}_{2}<=2.0000$

$0.0000<=\mathrm{V}<=2.0000$

$0.0000<=\eta<=1.0000$

Using Genetic algorithm, a set of solutions of dual problem (17) is given in the form of table in Table 2.

Table 2: Dual Solution

\begin{tabular}{cccc}
\hline $\mathbf{H}$ & $\boldsymbol{u}_{\mathbf{1}}$ & $\boldsymbol{u}_{\mathbf{2}}$ & $\mathbf{V}$ \\
\hline $\mathbf{0 . 9 5 6}$ & 1.863 & 1.961 & 1.844 \\
$\mathbf{0 . 9 5 6}$ & 1.867 & 1.607 & 0.956 \\
$\mathbf{1 . 0 0 0}$ & 1.836 & 0.741 & 1.273 \\
$\mathbf{0 . 9 7 9}$ & 0.716 & 1.118 & 1.994 \\
$\mathbf{0 . 9 5 3}$ & 1.935 & 1.075 & 1.894 \\
\hline
\end{tabular}

From the above table, we can clearly see that the solution obtained satisfies the fuzzy constraints as well as the fuzzy objective in both primal and dual problem. Also, it has been observed that difference between the tolerance limit and aspiration level is less. In other words, the decision makers have been provided with enough flexibility to choose satisfying solutions that maximize or minimize their utility functions. Genetic Algorithm gives multiple pareto optimal solutions in one single run. Therefore, it provides set of solutions to the decision maker. The following graph clearly shows comparison between the primal and dual objective function of different sets of solutions. 
International Journal of Soft Computing, Mathematics and Control (IJSCMC), Vol.4, No. 1, February 2015

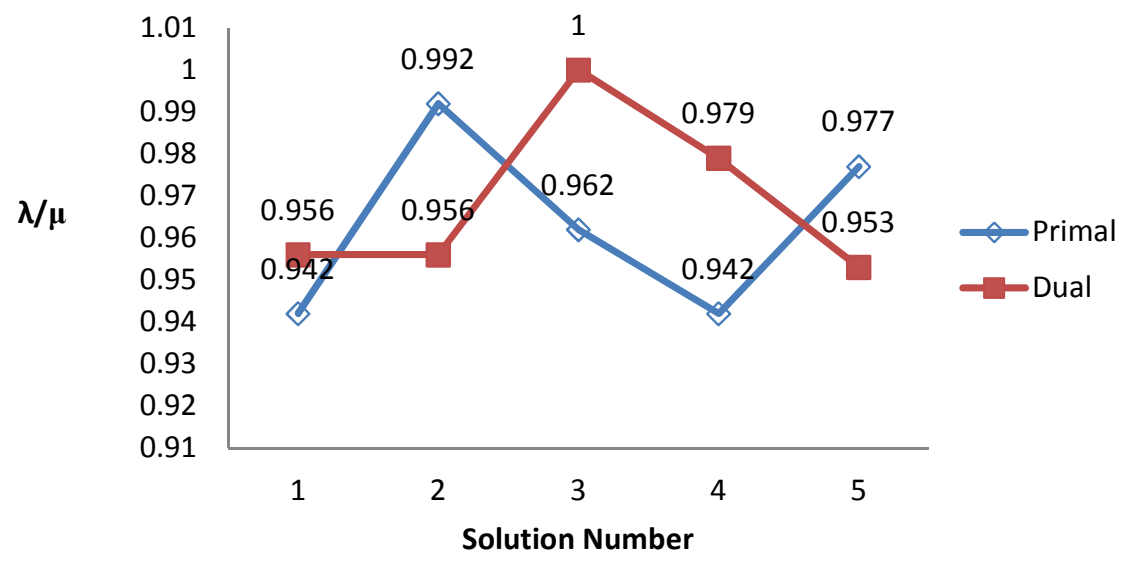

Figure 1: Primal and Dual objective function of different solution using GA

\section{CONCLUSIONS}

In this paper, an approach has been presented for a specific kind of fuzzy nonlinear fractional programming problem by constructing a pair of fuzzy non linear fractional primal and dual problems. Crisp form of the above primal and dual problems has been obtained by using exponential membership function. Duality results have been established to prove the duality relationship between above primal and dual problem and are illustrated by an example. The duality results fully satisfy the aspiration levels or the tolerance levels of the objective functions and the system constraints made by the decision maker. The difference between the achieved level and the allowable limit of the satisfying solutions of the decision maker is very less. The numerical example has also been solved by Genetic Algorithm Approach. Genetic Algorithm gives multiple pareto optimal solution. The decision maker can choose any optimal solution according to the convenience. The results of the present paper encourage us to apply duality results in variety number of fields of optimization problem.

\section{REFERENCES}

[1] Bellman, R. E., Zadeh, L. A., (1970) Decision Making in a Fuzzy Environment, Management Science, 17, 141-164.

[2] Bector, C. R. and Chandra, S., (2002) On duality in linear programming under fuzzy environment, Fuzzy Sets and Systems, 125, 317-325.

[3] Biswas, A. and Bose, K., (2012), Application of Fuzzy Programming Method for Solving Nonlinear Fractional Programming Problems with Fuzzy Parameters, Mathematical Modelling and Scientific Computation Communications in Computer and Information Science 283 104-113.

[4] Borza, M., Rambely, A. S. and Saraj, M., (2012) Solving linear fractional programming problems with interval coefficients in the objective function. A New Approach, Applied Mathematical Sciences, 6, 69, $3443-3452$.

[5] Chakraborty, M., Gupta, S., (2002) Fuzzy Mathematical Programming for Multi-objective Linear Fractional Programming Problem, Fuzzy Sets and Systems, 125, 335-342. 
International Journal of Soft Computing, Mathematics and Control (IJSCMC), Vol.4, No. 1, February 2015

[6] Chakraborty, A., Tiwari, S. P., Chattopadhyay, A. and Chatterjee, K. (2014), Duality in Fuzzy Multi objective linear programming with multi constraint, International Journal of Mathematics in Operations Research, Vol. 6, No. 3, pp. 297-315.

[7] Gupta, P. and Mehlawat, M. K., (2009) Duality for a convex fractional programming under fuzzy environment, International Journal of Optimization: Theory Methods and Applications, 1, 3, 291-301.

[8] Gupta, P. and Mehlawat, M. K., (2009), Bector- Chandra type duality in fuzzy linear programming with exponential membership functions, Fuzzy Sets and Systems, 160, 22, 3290-3308.

[9] Jameel, A. F., Sadhegi, A., (2012), Solving Nonlinear Programming Problem in Fuzzy Environment, Int. J. Contemp. Math. Sciences 7, 4, $159-170$.

[10] Jimenez, F., Sanchez, G., Cadenas, J. M., Gomez-Skarmeta, A. F., Verdegay, J. L., (2005), Computational Intelligence, Theory and Applications, Advances in Soft Computing, 33, 713-722.

[11] Kanaya, Z. A. (2010) An Interactive Method for Fuzzy Multiobjective Nonlinear Programming Problems, JKAU: Sci. 221 103-112; DOI: 10.4197 / Sci. 22-1.8.

[12] Lasdon, L. S, Waren, A. D., (1980), Feature Article: Survey of Nonlinear Programming Applications, Operations Research 28(5): 1029-1073.

[13] Liu, Y. J., Shi, Y. and Liu, Y. H. (1995) Duality of fuzzy MC2 linear programming: a constructive approach, J. Math. Anal. Appl., 194, 389-413.

[14] Rodder, W. and Zimmermann, H. J. (1980), Duality in fuzzy linear programming. In: (A. V. Fiacco, K. O. Kortane K Eds.). External methods and System Analysis. Berlin, New York, 415-429.

[15] Saad, O. M., M. S. Biltagy and T. B. Farag, An algorithm for Multi objective Integer nonlinear fractional programming problem under fuzziness, Gen. Math. Notes, 21 (2011) 1-17.

[16] Schaible, S., (1981) Fractional programming: applications and algorithms, European Journal of Operational Research, 7, 2, 111-120.

[17] Schaible, S., (1995), Fractional programming, in R. Horst and P.M. Pardalos (eds.), Handbook of Global Optimization, Kluwer Academic Publishers, Dordrecht-Boston-London, 495-608.

[18] Schaible, S. and Ibaraki, T., (1983), Fractional programming, European Journal of Operational Research 12, 3, 325-338.

[19] Stancu-Minasian, I. M., Pop, B., (1978) On a Fuzzy Set Approach to Solving Multiple Objective Linear Fractional Programming Problem, Fuzzy Sets and Systems, 134, 397-405.

[20] Zimmermann, H. J., (1978) Fuzzy programming and linear programming with several objective functions, Fuzzy Sets and Systems, 1, 45-55.

\section{Authors}

Ananya Chakraborty is Assistant Professor in the Department of Mathematics of Vemana Institute of Technology, Bangalore, India. She has done her Ph.D from Indian School of Mines, Dhanbad, Jharkhand, India. She is having almost 10 years of research experience. She has published many papers in national/ international journals. She has presented many papers in different conferences. Her current research interest includes Fuzzy programming, Operations Research. She is also a member of International association of computer science and information technology 